\title{
FT-NIR and Confocal Microscope Raman Spectroscopic Studies of Sesame Oil Adulteration
}

\author{
Jun Luo ${ }^{1, *}$, Tao Liu ${ }^{2}$, and Yande $\mathrm{Liu}^{2}$ \\ ${ }^{1}$ School of Mechanical Engineering, Jingdezhen Ceramic Institute, \\ Jingdezhen Jiangxi 333403 China \\ ${ }^{2}$ School of Mechanical and Electronical Engineering, \\ East China Jiaotong University, Nanchang 330013, P.R. China \\ luolitian@qq.com, jxliuyda163.com
}

\begin{abstract}
Adulteration of edible vegetable oils is a very serious problem affecting its commercial value and customers' health. FT-NIR and Confocal Micro-Raman have been explored for discriminating adulteration of sesame oils. The soybean, corn, and peanut oil were mixed into sesame oils in the range of 5 20\% (v/v),respectively, and their Confocal Micro-Raman and FT-NIR spectra were collected. Principal component analysis (PCA) and Partial least squares discriminant analysis (PLSDA) were used for the discrimination and classification of adulteration in sesame oil based on spectral data. In addition, the spectra were subjected to the pretreatements (eg, Savitzky-Golay smoothing, Multiplicatnive Scatter Correction, first derivatives and second derivatives) before developing PCA and PLS-DA models. FT-NIR was found to give better efficient in classification of adulteration oils by using PLS-DA with ca.100\% classification accuracy, and Raman also gave 100\% classification accuracy. The results demonstrated that FT-NIR and Confocal Micro-Raman both can be used to determine the authenticity of edible oils rapidlly.
\end{abstract}

Keywords: FT-NIR, Confocal Micro-Raman, Sesame Oil, Adulteration, PCA, PLSDA.

\section{Introduction}

As is well known, authenticity is a very important quality criterion for edible oils, and adulteration is a major problem for the specialist food markets. Many edible oils maybe become polluted through careless handling, or through unintentional or deliberate intervention. Many expensive oils are adulterated with cheaper alternatives to increase profit margins, or expensive ingredients are replaced with a cheaper alternatives. In some cases, this action simply means reducing the quality of the product, but such practices may even have moral and ethical implications. Numerous methods for assessing the authenticity of edible oils have been proposed, such as pyrolysis-mass spectrometry and gas chromatography[1], focused on the existence or

${ }^{*}$ Corresponding author.

D. Li and Y. Chen (Eds.): CCTA 2011, Part II, IFIP AICT 369, pp. 24-31, 2012.

(C) IFIP International Federation for Information Processing 2012 
absence of certain chemical compounds in the adulterated sample. However, the traditional methods for determination of authenticity based on titration are expensive, complex, laborious, time-consuming, require skilled personnel and sophisticated instrument capability. So, there is a need to develop a simple, cheap, and rapid alternative method to determine adulterants.

NIR spectra assesses chemical structures through the analysis of the molecular bands in the NIR spectrum (eg.C-H)[2], but the peaks in the near-infrared (NIR) region $\left(1,100 \sim 2,500 \mathrm{~nm}\right.$ or $\left.9,091 \sim 4,000 \mathrm{~cm}^{-1}\right)$ are broad and weak due to the overtone and combination vibrations originated from the chemical composition of samples. NIR spectroscopy was the first applied to determine the presence of adulterants (corn oil, sunflower oil, and raw olive residue oil) in olive oils[3].Kasemsumran, Sumaporn[4] made a new processing based on partial least squares (PLS) algorithm for the discrimination and determination of adulterants in pure olive oil using nearinfrared (NIR) spectroscopy. Balabin,RomanM.[5] tried to evaluate the efficiency of different methods for motor oils classification by using near-infrared (NIR) spectroscopy and SVM and PNN chemometric techniques were found to be the most effective ones for classification of motor oil.

The potential of Raman spectroscopy for the analysis of oils has been recognized for some decades. Raman spectroscopy has already been successfully used to determine important composition or physical structure parameters in a wide range of lipid sample types. Ming-Qiang Zou[6]established a method based on Raman spectroscopy vibration bands to authenticate genuine/fake olive oil and this method can reliably distinguish the genuine olive oils from the olive oils containing $5 \%$ (volume percentage) or more of other edible oils, such as soybean oil. Ru-Hui Weng[7] determined the authenticity of Camellia oleifera Abel Oil by Near Infrared Fourier Transform Raman Spectroscopy and a linear relationship with a high correlation coefficient $\left(\mathrm{R}^{2}=0.9938\right)$ was obtained. Rasha M. El-Abassy[8] used a visible Raman spectroscopic method for determining the free fatty acid (FFA) content of extra virgin olive oil with the aid of multivariate analysis.

This work shows the results of using Confocal Micro-Raman and FT-NIR spectroscopy combined with principal component analysis (PCA) and partial least squares discriminant analysis (PLS-DA) to detect the classification of adulteration in sesame oil and compares the performance of two spectroscopic techniques.

\section{Materials and Methods}

\subsection{Sample Description}

The samples used in this investigation were sesame (Shenzhen, Jinlongyu brand), soybean (Shenzhen,jinlongyu brand), corn (Shanxi, Xiwang brand) and peanut oil (Shenzhen,jinlongyu brand) that obtained from a local supermarkets. The concentration of adulteration was in the range of $5 \sim 20 \%$ by weight respectively, and the same was repeated with each concentration. All weighing was done by use of a analytic balance AUX120 (SHIMADZU, Germany) with the accuracy of $0.0001 \mathrm{~g}$. Totally, 66 adulterations of sesame oil mixed with soybean oil $(n=22)$, corn oil $(n=22)$ or peanut oil $(n=22)$ were prepared, and their spectra were collected. 


\section{$2.2 \quad$ FT-NIR Analysis}

The NIR measurements were performed using transmittance mode in the region of $12,000 \sim 4,000 \mathrm{~cm}^{-1}$ by means of TENSOR37 FT-NIR spectrometer(Bruker Optics Inc Germany), equipped with the RT-InGaAs detector. All the spectral data were collected with a $4 \mathrm{~cm}^{-1}$ spectral resolution and a total of 32 scans were co-added to ensure a sufficient signal-to-noise ratio. The oil samples were scanned in a rectangular quartz cuvettes. The quartz cuvette was cleaned with pure ethyl alcohol after successive measurements and dried to ensure the best possible sample spectra. To avoid any spectral variation due to some factors, air background spectrum was collected before each sample spectrum.

\subsection{Raman Analysis}

The Confocal Micro-Raman Spectrometer (BRUKER, SENTERRA) combined with a spectrometer module and a microscope module was used in this experiment. The spectrometer module was equipped with a CCD detector (thermo-electrically cooled, $1025 \times 256$ pixel) and a semiconductor laser operating at of $785 \mathrm{~nm}$ excitation $(50 \mathrm{~mW}$ at sample). The total spectral range $\left(90 \sim 3,500 \mathrm{~cm}^{-1}\right)$ was recorded within $15 \mathrm{~s}$ of total integration time at $9 \mathrm{~cm}^{-1}$ resolution and every measurement consisted of five averaged signal accumulations each with an actual exposure time of $3 \mathrm{~s}$. The oil samples analyzed were poured into a silver spoon and placed on the sample stage (motorized stage $100 \mathrm{~mm} \times 80 \mathrm{~mm}$ ).

\subsection{Discriminant Analysis}

The OPUS software (verion. 6.5; Bruker Optik GmbH, Germany) was employed for the spectral data collection. PCA and PLS-DA were performed by using the Unscrambler software (verion. 10.1; CAMO AS, Trondheim, Norway). In order to minimize the effect of light scattering, The spectra were subjected to Savitzky-Golay smoothing (7-point, 2orders), Multiplicative Scatter Correction, first derivatives (7-point Savitsky-Golay filter) and second derivatives (7-point Savitsky-Golay filter) before developing PCA and PLS-DA models for the discrimination. For the PLS-DA analysis, a set of values were given to the spectra of examined adulterant arbitrarily for the dummy variable "species", such as corn=1.0, soybean=2.0, peanut=3.0. All samples with an error around predicted value more than or equal to 0.5 were classified as not being members of the examined adulterant type. On the other hand, those with an error value lower than 0.5 were classified as being members of the adulterant type. The results are presented as percentage, the expression is given as correct discrimination rate $(C D R)$ of models.

$$
C D R=\frac{N-n}{N}
$$

where $N$ represents total number of calibration (validation)samples for each model, $n$ is the number of erroneous judgement of samples in every model.

Principal component analysis (PCA) is a nondirected, nonsupervised method of data reduction, which determines the main sources of variation within the spectra, 
allowing transformation of several related data points into a single number[9]. Discriminant analysis is a supervised classification technique where the number of groups and the samples that belong to each group are previously defined [10]. Partial least squares discriminant analysis (PLS-DA) differs from PCA in that each source of variation is not ranked in relation to the overall variation, but according to how it accounts for variation between data subsets(groups).

For chemometric analysis, five replications of each sample were used for calibration and validation models. A total of 110 spectra of NIR or Raman were acquired for each adulterant. The same sets of samples were used for enabling comparison of the spectral techniques. For each sample, four of 5 measurements were used for the development of calibration model and rest for validation. Hence a total of 264 individual samples were used for calibration and 66 for validation.

\section{$3 \quad$ Results and Discussion}

As we know, every oil differs in composition, length and unsaturated degree of the fatty acids as well as their positions in the chain. Raman spectra represent a combined fingerprint pattern unique to each oil and are used for discriminant analysis. Many analysis were conducted using regions specific to the $\mathrm{C}=\mathrm{C}$ bond vibration. The double bond considered as an unsaturated bond and the intensities, areas or heights of its peak in this region might indicate the degree of unsaturation in fatty acids, sterols, and vitamins.

\subsection{FT-NIR Spectra}

Fig. 1 shows some FT-NIR spectra of sesame oil adulterated with corn oil、soybean oil and peanut oil in the $12,000 \sim 4,500 \mathrm{~cm}^{-1}$ region. The spectra shows three major band groups at around 8,263,7,186, and 5,788 $\mathrm{cm}^{-1}$. The region between 5,500 and $6,000 \mathrm{~cm}^{-1}$ is the first overtone of the $\mathrm{C}-\mathrm{H}$ stretching from- $-\mathrm{CH}_{2},-\mathrm{CH}_{3}$ and $-\mathrm{CH}=\mathrm{CH}-$ functional groups. The region between 7,000 7,400 $\mathrm{cm}^{-1}$ contains bands assigned to the combination modes of the $\mathrm{CH}$ stretching and $\mathrm{CH}$ deformation vibration modes. The region between $8,000-8,500 \mathrm{~cm}^{-1}$ consists of bands due to the second overtone of $\mathrm{CH}$ stretching modes of the $\mathrm{CH}_{3}$ and $\mathrm{CH}_{2}$ groups stretching modes. It is noted in Fig. 1 that those mean NIR spectra are very similar to each other, and it is not easy to classify the spectra into each adulterant type. Therefore, the PCA and PLS-DA method are applied in the study to identify the unknown adulterations in sesame oil samples.

An overall PCA was carried out using all samples of each oil. Fig 2 shows the classification results of the PCA on data sets containing three kinds of the oil spectra in score plots. The PCA model was achieved using 1PCs and explaining 97\% of variance in the spectral data. The plots shows that the peanut oil is clearly separated from the others. But corn oil has so many portion in common with soybean oil, which gives no boundaries. As with all non-supervised methods for classification, the plot built by PCA reveals and explains the patterns of the samples studied, but the assignation of new samples to a class may be a difficult task, as the method does not calculate a rule to generate boundaries or regions for the groups of samples obtained. Hence, supervised method (PLS-DA) was introduced in order to improve the results. 


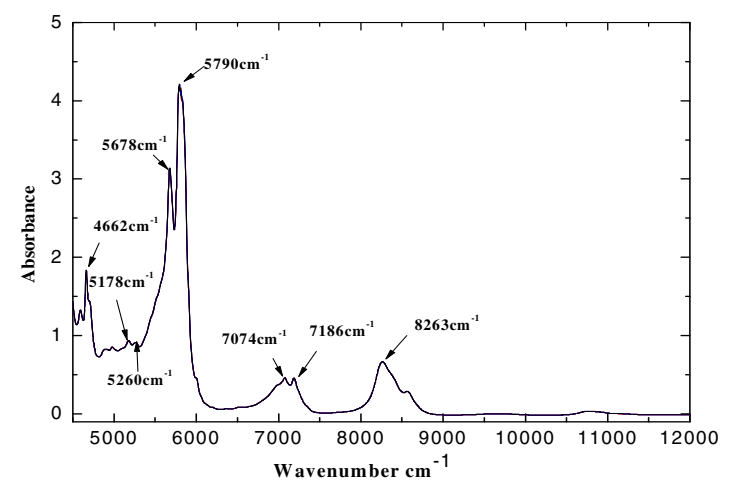

Fig. 1. FT-NIR spectra in the $12,000 \sim 4,500 \mathrm{~cm}^{-1}$ region of sesame oil with adulteration

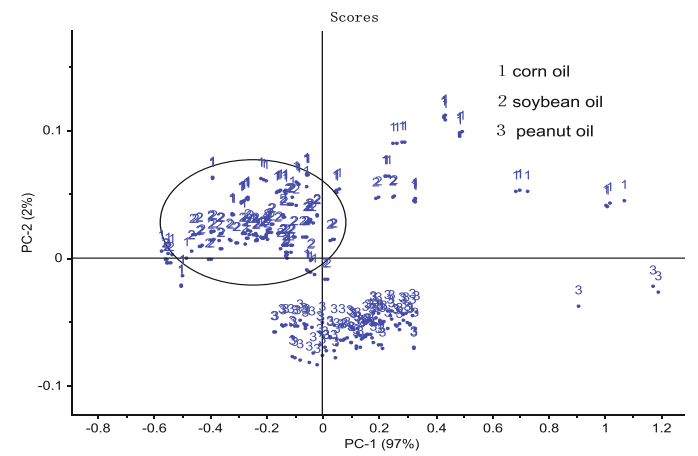

Fig. 2. PCA plots for classification of oils using FT-NIR spectra

The statistical results of PLS-DA based on FT-NIR spectra are summarized in Table 1. The discrimination models are developed by using two selected spectral ranges and five spectra types: original and four pretreatment spectra. The rate of correct classification obtained from discriminant analysis of the spectra between $6,410.4$ and $8,917.4 \mathrm{~cm}^{-1}$ is found to be better than the data from the spectral region between 4,000 and $12,000 \mathrm{~cm}^{-1}$. In the region $6,410.4 \sim 8,917.4 \mathrm{~cm}^{-1}$, the models using any pretreatment yield $100 \%$ correct classification of the prediction set $(\% \mathrm{CP})$, especially the second derivative spectra with only the lowest number of PLS factor 2. As is well-known, a small number of PLS factor required for developing a model that provides acceptable results indicates the stability and accuracy of such model[11]. However, when the wide spectral range $\left(4,000 \sim 12,000 \mathrm{~cm}^{-1}\right)$ is used, the model developed by using the second derivative spectra shows the worst classification performance. This is because the wide spectral range includes a region that contains more noise than relevant information to the model development for the concentration of an analyte. Therefore, the optimal models could be selected from the models giving the best predictive accuracy with the smallest number of PLS factor 
Table 1. Discrimination analysis of FT-NIR spectra of the adulterant types in sesame oil

\begin{tabular}{ccccc}
\hline & pretreatment & Factors & \% CDRC & \% CDRV $^{\mathrm{b}}$ \\
\hline $6,410.4-$ & None & 6 & 100 & 100 \\
$8,917.4 \mathrm{~cm}^{-1}$ & smoothing & 6 & 100 & 100 \\
& MSC & 4 & 100 & 100 \\
& 1 st & 3 & 100 & 100 \\
$4,000-$ & 2nd & 2 & 100 & 100 \\
$12,000 \mathrm{~cm}^{-1}$ & None & 10 & 99.6 & 90.9 \\
& smoothing & 10 & 99.6 & 89.4 \\
& MSC & 10 & 99.6 & 95.5 \\
& 1st & 4 & 86.4 & 63.6 \\
& 2nd & 5 & 41.7 & 45.5 \\
\hline
\end{tabular}

$\mathrm{CDRC}^{\mathrm{a}}=$ correct discrimination rate of calibration, $\mathrm{CDRV}^{\mathrm{b}}=$ correct discrimination rate of validation.

\subsection{Confocal Micro-Raman Spectra}

The Confocal Micro-Raman spectra of pure sesame oil are shown in Fig 3. The Fig 3 shows that many major bands of the oleic acid were detected in the Raman spectra of the oil samples, and are attributed to the main components in the oil, which are fatty acids. It should be noted that the region between 1,600 and $1,700 \mathrm{~cm}^{-1}$ contains the stretching vibration of the $C=C$ bond which is very distinctive around $1,655 \mathrm{~cm}^{-1}$ in Raman spectra. It demonstrates that spectra can display non-polar groups of samples which can not be observed in the FT-NIR spectra.

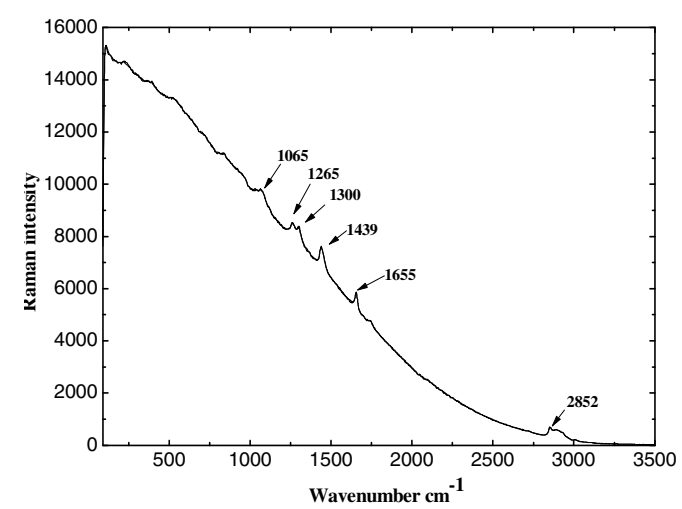

Fig. 3. Raman spectra of pure sesame oil in the $90 \sim 3,500 \mathrm{~cm}^{-1}$ region

According to Fig 4, we can see that the discriminant models built with Raman spectra by PCA are the same weak to decide for samples as the models built with FTNIR spectra. Although corn oil can be separated from others successfully, giving a value of good prediction 100\%, Soybean and peanut oil are both distributed in the same circle. Therefore, we need better methods to make models perfect. 


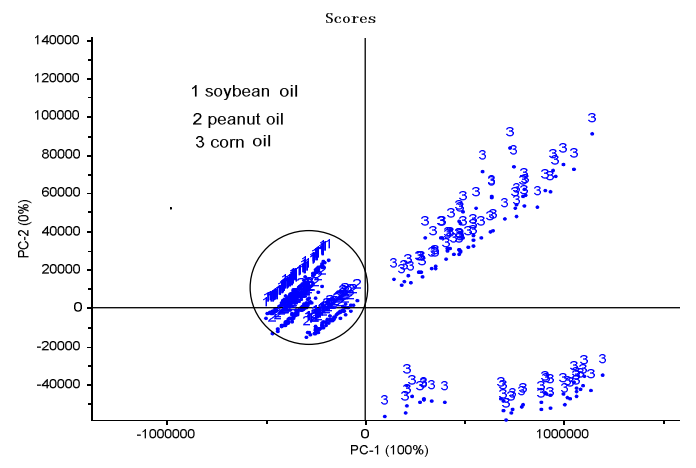

Fig. 4. PCA plots for classification of oils using Raman

The spectra regions $\left(90 \sim 1,795,2,818.5 \sim 3,159\right.$ and $\left.90 \sim 3,500 \mathrm{~cm}^{-1}\right)$ were used for discriminant analysis. The results obtained from PLS-DA for the adulteration discrimination in sesame oil are summarized in Table 2. The rate for correct classification with PLS-DA methods was found to be more than $95.5 \%$ for both the calibrationand validation samples due to the degree of unsaturation. It is noted in Table 4 that the excellent prediction results are obtained mostly from the models built with the regions of $90 \sim 1,795$ and $2,818.5 \sim 3,159 \mathrm{~cm}^{-1}$ at the same time. It also can be seen that when the spectral data are pretreated by MSC, the model gives the highest rate $(100 \%)$ for correct classification. However, the results from Confocal MicroRaman measurements were not as good as these from FT-NIR. Vibrations from polar groups, such as $\mathrm{C}=\mathrm{O}$ and $\mathrm{O}-\mathrm{H}$, are very weak in Raman spectra, which result in the missing of some important vibrations and these vibrations maybe play very important roles in discriminant analysis.

Table 2. Discrimination analysis of Raman spectra of the adulterant types in sesame oil

\begin{tabular}{ccccc}
\hline & pretreatment & Factors & \%CDRC & \%CDRV $^{\mathrm{b}}$ \\
\hline $90 \sim 1,795$ & None & 10 & 100 & 96.9 \\
$2,818.5 \sim 3,159 \mathrm{~cm}^{-1}$ & smoothing & 9 & 99.2 & 97.0 \\
& MSC & 10 & 100 & 100 \\
& $1 \mathrm{st}$ & 5 & 96.7 & 96.7 \\
$90 \sim 3,500 \mathrm{~cm}^{-1}$ & 2nd & 7 & 100 & 97.0 \\
& None & 11 & 100 & 98.5 \\
& smoothing & 10 & 99.2 & 98.5 \\
& MSC & 9 & 99.6 & 100 \\
& 1st & 6 & 96.2 & 95.5 \\
& 2nd & 5 & 99.2 & 95.5 \\
\hline
\end{tabular}

$\mathrm{CDRC}^{\mathrm{a}}=$ correct discrimination rate of calibration, $\mathrm{CDRV}^{\mathrm{b}}=$ correct discrimination rate of validation. 


\section{Conclusions}

This study compared Confocal Micro-Raman and FT-NIR spectroscopy for detecting the classification of adulteration in sesame oil. The results revealed that either of the spectral methods is easier to perform and the FT-NIR method was found to be more efficient. However, Raman spectroscopy techniques could provide exquisite structural insights into functional groups of oils for discriminant analysis. In addition, it is clear from the results above section that the PLS-DA is a very powerful method to identify adulterant types in the adulteration of sesame oil samples.

Acknowledgements. The authors gratefully acknowledge the financial support provided by National Science and Technology Support Program (31160250,61178036), Natural Science Foundation of Jiangxi Province (2008GQN0029, 2007GZN0266), Special Science and Technology-Support Program for Foreign Science and Technology Cooperation Plan (2009BHB15200), Technological expertise and academic leaders training program of Jiangxi Province (2009DD00700).

\section{References}

1. Yang, Z., et al.: Oil fingerprinting analysis using commercial solid phase extraction (SPE) cartridge and gas chromatography-mass spectrometry (GC-MS). Anal. Methods 3, 628-635 (2011)

2. Cozzolino, D., Fassio, A., et al.: Verification of silage type using near-infrared spectroscopy combined with multivariate analysis. Journal of Agricultural and Food Chemistry 56, 9-83 (2008)

3. Wesley, I.J., Barnes, R.J., et al.: Measurement of Adulteration of Olive Oils by NearInfrared Spectroscopy. J. Am. Oil Chem. Soc. 72, 289-292 (1995)

4. Kasemsumran, S., et al.: Partial Least Squares Processing of Near-InfraredSpectra for Discrimination and Quantification of Adulterated Olive Oils. Spectroscopy Letters 38(6), 839-851 (2005)

5. Balabin, R.M., Safieva, R.Z., et al.: Near-infrared (NIR) spectroscopy for motor oil classification: From discriminant analysis to support vector machines. Microchemical Journal 98(1), 121-128 (2011)

6. Zou, M.-Q., Zhang, X.-F., et al.: Rapid Authentication of Olive Oil Adulteration by Raman Spectrometry. J. Agric. Food Chem. 57(14), 6001-6006 (2009)

7. Weng, R.-H., Weng, Y.-M., Chen, W.-L.: Authentication of Camellia oleifera Abel Oil by Near Infrared Fourier Transform Raman Spectroscopy. Journal of the Chinese Chemical Society 53, 597-603 (2006)

8. El-Abassy, R.M., Donfack, P., Materny, A.: Rapid Determination of Free Fatty Acid in Extra Virgin Olive Oil by Raman Spectroscopy and Multivariate Analysis. J. Am. Oil Chem. Soc. 86, 507-511 (2009)

9. Renwick Beattie, J., Steven, E.J., et al.: Classification of Adipose Tissue Species using Raman Spectroscopy. Lipids 42, 679-685 (2007)

10. Cozzolino, D., Fassio, A., et al.: Verification of silage type using near-infrared spectroscopy combined with multivariate analysis. Journal of Agricultural and Food Chemistry 56, 79-83 (2008)

11. Martens, H., Næs, T.: Multivariate Calibration. John Wiley, Chichester (1989) 